\title{
Alles nur inszeniert? Das politische Kino Jafar Panahis
}

„Sie machen doch hier einen Film, oder?“, fragt in Jafar Panahis 2015 erschienenem Film TAXI der Fahrgast Omid den merkwürdigen Taxifahrer, in dessen Wagen er gestiegen ist und hinter dem er - zu Recht, wie sich herausstellt - den iranischen Regisseur Panahi vermutet. ${ }^{1}$ In diesem Taxi spielt sich Alltägliches und zugleich Sonderbares ab, sodass Omid sich bald als Teil einer filmischen Inszenierung wähnt, die Panahi, der Regisseur (nicht der Taxifahrer), bewusst so angeordnet hat. „Sie haben das alles nur inszeniert und denken, ich falle darauf herein“ (TAXI, 00:17:59), glaubt Omid zu wissen - und ist sich bis zum Ende seiner Taxifahrt so sicher doch nicht. Mit Omid, der mit seiner Skepsis die vierte Wand durchbricht, fragen sich auch die Filmzuschauer*innen sukzessive: „Ist das hier alles nur inszeniert?“ Wo endet die Dokumentation, wo die Fiktion - oder ist die Grenze zwischen beiden längst aufgelöst?

Jafar Panahi ist ein in Ost-Aserbaidschan geborener iranischer Filmemacher, der bereits in frühester Jugend eine Leidenschaft für das Kino, insbesondere für das italienische Kino der 1950er Jahre entwickelt. Als Assistent seines Lehrmeisters und späteren Mentors Abbas Kiarostami lernt er ein iranisches Kino kennen, das ein Weltkino ist: Kiarostami, 2016 verstorben, gilt heute als einer der wichtigsten Regisseure der globalen Filmgeschichte. ${ }^{2}$ Er ist es auch, der das Drehbuch zu Panahis Spielfilmdebüt BADKONAKE SEFID (dt. DER WEISSE BALLON) ${ }^{3}$ verfasst. Der Film wird 1995 sogleich mit der Goldenen Kamera der Filmfestspiele von Cannes ausgezeichnet. Seither ist Panahi zu einem der bekanntesten Filmemacher^innen des Iran aufgestiegen, hat 2000 mit dem Spielfilm DAYEREH (dt. DER KREIS) den Goldenen Löwen in Venedig und mit OfFsIDE 2006 den Silbernen Bären der Berlinale gewinnen können. OFFSIDE, ein Film über eine Gruppe von Mädchen und Frauen, die versuchen, das entscheidende WM-Qualifikationsspiel der iranischen Fußballnationalmannschaft in Teheran zu besuchen, ist vielleicht der bekannteste Film des Regisseurs: im Iran verboten - in Europa gefeiert. Leicht hatte es

1 TAXI. Reg. Jafar Panahi. Iran 2015, TC: 00:10:12. Der Film wird im Folgenden im Fließtext mit Timecode-Angabe und seinen deutschen Untertiteln zitiert.

2 Vgl. den Nachruf von Nicodemus, Katja. „Der Freie“. In: Die Zeit, Nr. 29 vom 07.07.2006. https: //www.zeit.de/2016/29/nachruf-abbas-kiarostami (24.03.2020).

3 Badkonake Sefid/Der weisse Ballon. Reg. Jafar Panahi. Iran 1995. 
Panahi mit seinen tabubrechenden Filmen im Iran nie, im Jahr 2010 wurde er schließlich festgenommen und inhaftiert. Obgleich vor der Gerichtsverhandlung zunächst eine temporäre Freilassung erfolgte, wurde er im Anschluss zu sechs Jahren Haft und einem 20-jährigen Berufs- und Ausreiseverbot verurteilt. Seither steht Panahi unter einer Art Hausarrest, jeder seiner Schritte wird überwacht und die angekündigte, aber noch nicht in Kraft getretene Haftstrafe ist das Damoklesschwert, mit dem der Regisseur (wie viele andere seiner Kolleginnen und Kollegen) lebt. Trotz des Berufsverbots hat Panahi seit 2010 heimlich und illegalerweise vier Filme drehen können - unter widrigsten Produktionsbedingungen, die diese Filme inhaltlich wie formal-technisch durchaus sichtbar machen.

\section{Authentizität der Bilder}

Außerfilmische Realität und filmische Fiktion verschwimmen in Panahis Filmen konsequent - ganz unabhängig von ihrer Markierung als feature film, documentary oder docufiction. Programmatisch ist diesbezüglich schon sein zweiter, 1997 erschienener Film AyNEH (dt. DER SPIEGEL), der vordergründig von einem kleinen Mädchen erzählt, das beschließt, allein durch Teheran den Weg nach Hause zu finden, nachdem seine Mutter vergessen hat, es pünktlich von der Schule abzuholen. Die filmische Fiktion bricht hier abrupt ab, wenn die Kinderschauspielerin Mina aus ihrer gleichnamigen Rolle fällt, in die Kamera schaut und trotzig ankündigt, den Film nicht mehr weiter drehen zu wollen und stattdessen nach Hause zu gehen: „I'm not acting anymore!“4

Panahi aber lässt die Kamera weiterlaufen: Aus dem Spielfilm (über ein Mädchen, das sich allein durch die Großstadt schlägt) wird plötzlich ein Dokumen-

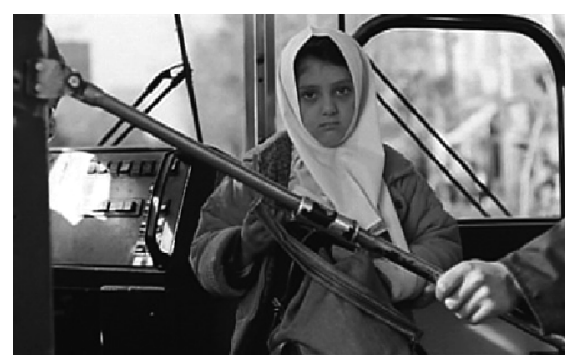

Abb. 1: Ayneh. Reg. Jafar Panahi. Iran 1997, TC: 00:38:49.

4 AYNeH/Der Spiegel. Reg. Jafar Panahi. Iran 1997, TC: 38:50:00. 
tarfilm (über ein Mädchen, das sich allein durch die Großstadt schlägt) - oder ist auch das alles nur inszeniert?

Beinahe sämtliche Filme Panahis bewegen sich an der Grenze zwischen den Gattungen Spielfilm und Dokumentation und machen eine klare Unterscheidung von afilmischer und profilmischer Referenzialisierung im Sinne Etienne Souriaus nahezu unmöglich. ${ }^{5}$ Afilmisch bezeichnet mit Souriau jene physische Wirklichkeit, die unabhängig von der filmischen Inszenierung, allem voran der Mise-enscène, besteht. Profilmisch hingegen bezeichnet jene physikalisch ebenfalls fassbaren, jedoch für den Film generierten bzw. arrangierten Gegenstände. Die afilmische Wirklichkeit wird etwa dort zur profilmischen, wo ein real existierendes Schloss zur Kulisse eines Märchenfilmes wird. Mit Blick auf die Differenzierung zwischen Dokumentar- und Spielfilm zeigt sich, so unterstreicht im Anschluss an Souriau der Medienwissenschaftler Florian Mundhenke, dass diese sich gerade in der „Schärfe und Deutlichkeit des Verweises auf das Afilmische“6 unterscheiden. Die Referenzialisierung auf das Afilmische sei beiden Filmgattungen durchaus möglich - der Spielfilm aber verzichte im Sinne eines Illusionsprozesses darauf, während Referenzialität im Dokumentarfilm, so Mundhenke, ,aktiv benutzt und immer wieder diskursiv eingeholt wird. " 7 Auch die Filme Panahis thematisieren ihre Referenzialität auf das Afilmische konsequent - und verweigern sich dort, wo sie die Wahrheit des Dargestellten hinterfragen, einer einheitlichen Semantisierung von Begriffen wie Wirklichkeit, Realismus oder Authentizität auf der inhaltlichen wie der formal-ästhetischen Ebene.

Der in Panahis Werk filmisch reflektierte Begriff der Authentizität verweist dabei auf die ambivalente erzähltheoretische Bewertung, die dieser in den letzten Jahren in narratologischen Auseinandersetzungen erfahren hat. Gehen Michael Scheffel und Matías Martínez in ihrer erzähltheoretischen Einführung noch von einer Gleichsetzung der Begriffe faktual und authentisch aus, ${ }^{8}$ macht es sich das Freiburger Graduiertenkolleg „Faktuales und Fiktionales Erzählen“ (GRK 1767) unter der Leitung Monika Fluderniks zur methodischen Grundvoraussetzung,

5 Vgl. Souriau, Etienne. „Die Struktur des filmischen Universums und das Vokabular der Filmologie“ [= La structure de l'univers filmique et le vocabulaire de la filmologie 1951]. In: montage AV. Zeitschrift für Theorie und Geschichte audiovisueller Kommunikation, 6.2 (1997), 140-157, hier 146-147.

6 Mundhenke, Florian. Zwischen Dokumentar- und Spielfilm. Zur Repräsentation und Rezeption von Hybrid-Formen. Wiesbaden: Springer 2017, 75.

7 Mundhenke: Zwischen Dokumentar- und Spielfilm, 75.

8 „Fiktional steht im Gegensatz zu ,faktual` bzw. ,authentisch“ und bezeichnet den pragmatischen Status einer Rede.“ Martínez, Matías/Scheffel, Michael. Einführung in die Erzähltheorie. 10., überarb. und aktual. Auflage. München: C.H.Beck 2016, 16. 
„dass man Faktualität nicht mit Begriffen wie Authentizität, Realismus oder Mimesis gleichsetzt“, sondern Effekte von Authentizität sowohl in faktuale wie fiktionale Erzählformen Eingang finden. ${ }^{9}$ Abhilfe angesichts dieser widersprüchlichen Begriffsdefinition verspricht jene Unterscheidung, die Antonius Weixler vorschlägt, wenn er eine referenzielle Auffassung von Authentizität abgelöst und durch eine relationale ersetzt sieht. ${ }^{10}$ Relationale Authentizität substituiert tradierte Formen der Referenz-Authentizität bzw. hinterfragt diese bereits: Die Frage nach der Wahrheit des Dargestellten wird - diese These Weixlers lässt sich auch für Panahis Filmpoetik fruchtbar machen - „durch die nach der Wahrhaftigkeit der Darstellung ersetzt. "11 Begreift man Wahrhaftigkeit im Sinne des von Jürgen Habermas definierten Begriffs der subjektiven Wahrhaftigkeit als einen Geltungsanspruch, der sich nicht auf die äußere Realität, sondern die Aufrichtigkeit der manifesten Sprechintention bezieht, ${ }^{12}$ sind Panahis Filme eben dort authentisch, wo ihre Figuren den politisch instrumentalisierten Begriff der Wahrheit geradezu herausfordern. Das ist, wie ich im Folgenden zeigen möchte, allen voran in dem dokufiktionalen Film TAXI (auch als TAXI TEHERAN vertrieben) der Fall - dessen Fiktion, wie es Thomas Klingenmaier in der Stuttgarter Zeitung formuliert, „ganz ins Licht der Wahrhaftigkeit getaucht ist“..13 Daran ändern die strengen Zensurmaßnahmen des iranischen Regimes nichts, im Gegenteil: Gerade dort, wo sich der Film auf die aus den politischen Zensurmaßnahmen resultierenden „Widerständigkeiten“ einlässt, erreicht er im Glücksfall, pointiert Christina Nord in ihrer Filmkritik ganz richtig, „besondere Formen von Wahrhaftigkeit." ${ }^{14}$ Dass ein Film, der sich nicht für eindeutige Zuschreibungen (Dokumentation oder Fiktion) entscheiden mag, an der Kategorie der Wahrhaftigkeit und damit der Authentizität festhält, lässt sich auch als film- wie fiktionstheoretisches Plädoyer für die Auf-

9 Fludernik, Monika/Falkenhayner, Nicole/Steiner, Julia. „Einleitung“. In: Faktuales und Fiktionales Erzählen. Schriftenreihe des Graduiertenkollegs 1767. Hg. Monika Fludernik, Nicole Falkenhayner, Julia Steiner. Würzburg: Ergon 2015, 7-22, hier 10.

10 Vgl. hierzu auch de Beitrag von Antonius Weixler in diesem Band.

11 Weixler, Antonius. „Authentisches erzählen - authentisches Erzählen. Über Authentizität als Zuschreibungsphänomen und Pakt“. In: Authentisches erzählen. Produktion, Narration, Rezeption. Hg. Antonius Weixler. Berlin/Boston: De Gruyter 2012, 1-32, hier 2.

12 Vgl. Habermas, Jürgen. „Erläuterungen zum Begriff des kommunikativen Handelns“. In: Vorstudien und Ergänzungen zur Theorie des kommunikativen Handelns. Hg. Jürgen Habermas. Frankfurt a. M.: Suhrkamp 1995 [1982], 571-606, hier 588.

13 Klingenmaier, Thomas. „Die Gewitztheit des Verfolgten“. In: Stuttgarter Zeitung vom 23. Juli 2015. https://www.stuttgarter-zeitung.de/inhalt.untergrundkino-aus-dem-iran-taxi-teherandie-gewitztheit-des-verfolgten.afd26a5a-6151-4e3b-b4bf-26c3eadc43ce.html (15.03.2020).

14 Nord, Christian. „Eine Irrfahrt ohne Abspann“. In: taz vom 22.7.2015. https://taz.de/FilmstartTaxi-Teheran/!5215755 (15.02.2020). 
hebung strikter Grenzen zwischen den Gattungen Dokumentar- und Spielfilm verstehen. Selbst der hybrid anmutende Begriff der Dokufiktion verspricht hier nur bedingt Abhilfe, da er als Wortzusammensetzung, wie Christian Hißnauer zu Recht bemerkt, tradierte Binarismen voraussetzt und letztlich „die Vorstellung von abgrenzbaren Kategorien als stummes Wissen“ reproduziere. ${ }^{15}$ Tatsächlich lassen sich das Dokumentarische und das Fiktionale kaum als Opposition begreifen, vielmehr seien beide, so Hißnauer, über das Merkmal des Narrativen verbunden: „Jede Erzählung bringt Fiktion ins Spiel.“16 Die theoretisch konstatierte Fiktionalität auch dokumentarischer Darstellungen ${ }^{17}$ ändert nichts daran, dass sich die Unterscheidung von Dokumentation und Fiktion im Alltag durchaus durchgesetzt hat und nicht zuletzt $\mathrm{zu}$ einer entscheidenden Kategorie im Filmvertrieb avanciert ist. Entsprechend begreift Hißnauer die Unterscheidung zwischen den Gattungen Spielfilm und Dokumentarfilm unter Rückgriff auf den semio-pragmatischen Ansatz Roger Odins ${ }^{18}$ nicht als Aussage über den Fiktionsgehalt der filmischen Bilder, sondern als Rezeptionsmodus. Der maßgebliche Unterschied zwischen der fiktivisierenden und der dokumentarisierenden Lektüre besteht dann (mit Hißnauer und Odin) darin, dass letztere eine real vorhandene Aussageinstanz, keineswegs aber die Realität des Dargestellten voraussetzt. ${ }^{19}$ Mit Hißnauer geht der vorliegende Beitrag von einer theoretisch kaum haltbaren Unterscheidung von fiktional und dokumentarisch aus, die gleichwohl als spezifische (und durch paratextuelle Lektüreanweisungen programmierbare) Form der Rezeption sozial relevant bleibt.

15 Hißnauer, Christian. „MöglichkeitsSPIELräume. Fiktion als dokumentarische Methode. Anmerkungen zur Semio-Pragmatik Fiktiver Dokumentationen“. In: MEDIENwissenschaft 1 (2010), 17-28, hier 17.

16 Hißnauer: MöglichkeitsSPIELräume, 18. Hißnauer verweist hier auf die einschlägige Dissertation von Hohenberger, Eva: Die Wirklichkeit des Films. Dokumentarfilm. Ethnographischer Film, Jean Rouch. Hildesheim/Zürich/New York: Olms 1988.

17 Vgl. Blum, Philipp. „Doku-Fiktionen. Filme auf der Grenze zwischen Fiktion und Non-Fiktion als ästhetische Interventionen der Gattungslogik“. In: MEDIENwissenschaft 2 (2013), 130-144, hier 132: „Dass die Grenze zwischen fiktional und faktual weder beständig noch stabil - geschweige denn ,natürlich' - ist, kann mittlerweile [...] als opinio communis gelten.“

18 Vgl. Odin, Roger. „Dokumentarischer Film - dokumentarisierende Lektüre“. In: Bilder des Wirklichen. Texte zur Theorie des Dokumentarfilms. Hg. Eva Hohenberger. 3. Auflage. Berlin: Vorwerk 8 2006, 259-275.

19 Vgl. Hißnauer: MöglichkeitsSPIELräume, 20. 


\section{Filmen in Gefangenschaft}

Die vier zuletzt veröffentlichten Filme Panahis, ThIS Is NOT A FILM (Iran 2011), Pardé/Closed CuRtain (Iran 2013), TAXI (Iran 2015) und SE RoKh/Drei GesichTER (Iran 2018), stellen die vermeintlich zwischen Dokumentation und Fiktion changierende Ästhetik seiner Filme exemplarisch aus. Das Besondere an diesen jeweils heimlich gedrehten und illegal ins Ausland geschafften Filmen ist, dass sich ihre hybride Filmsprache dort als explizit kritisches Moment versteht, wo sie eben auch den politischen Bedingungen geschuldet ist, unter denen sie entstehen müssen, nachdem das Berufsverbot ausgesprochen wurde. Formale Besonderheiten wie die Besetzung der Rollen mit (größtenteils befreundeten) Laiendarsteller`innen, eine oftmals natürliche Beleuchtung und die geringe Qualität der Tonspur sowie eine den finanziellen Engpässen geschuldete reduzierte Postproduktion gehören ebenso dazu wie filmische Inhalte, die der unmittelbaren Gegenwart Teherans oder gar Panahis Alltag entnommen sind und in meist geschlossenen Räumen sowie an Drehorten gefilmt werden, die abgelegen oder zumindest schwer einsehbar sind.

This Is Not a Film spiegelt Panahis Situation nach dem Berufsverbot wider und wurde als Dokumentarfilm rezipiert. Er feierte seine Premiere beim Film Festival in Cannes 2011, nachdem er auf einem USB-Stick gespeichert und in einem Kuchen versteckt außer Landes geschmuggelt worden war. Der Film zeigt den unter Hausarrest stehenden Panahi am Tag des Iranischen Neujahrsfestes Nouruz, wie er mit der Auflage, keine Filme mehr zu drehen, hadert und dabei von einem Freund, dem iranischen Dokumentarfilmer Mojtaba Mirtahmasb, gefilmt wird, oder wie er bisweilen selbst die Kamera auf sich richtet. Einen Tag lang erleben die Zuschauer^innen, wie Panahi durch die eigene Wohnung tigert, neue Filmprojekte andenkt und verwirft, abgedrehte Filme noch einmal prüft, um die Realität seines Arrests mit den Filmerzählungen abzugleichen. Verzweifelt mutet die im Film gezeigte dauernde Auseinandersetzung mit einem Identitätsentwurf an, der ohne den Film als Ausdrucksmedium nicht gelingen will - vielleicht am eindrucksvollsten vergegenwärtigt in jener Szene, in der Panahi versucht, im eigenen Wohnzimmer das fiktive Set eines Filmes nachzuvollziehen, den er ursprünglich drehen wollte (vgl. Abb. 2).

THIS Is Not A FILM will kein politisches Zeichen gegen das Berufsverbot setzen, sondern die existenzielle Identitätskrise spürbar machen, in die Panahi gerät, weil er mit der Kamera keine Geschichten mehr erzählen darf. In diesem vermeintlich dokumentarischen Film zeigt Panahi, dass das Dokumentarische ge- 

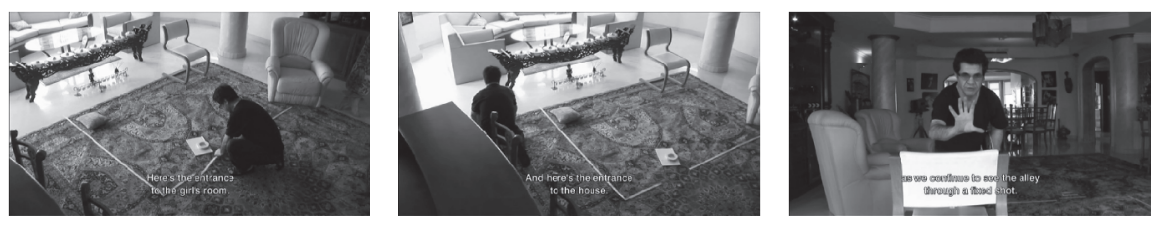

Abb. 2: ThIS IS Not A FILM. Reg.Jafar Panahi, Iran 2011, TC: 00:17:58ff.

rade nicht seine Erzählform ist. Eine selbstreferenzielle Passage des Films ${ }^{20}$ hält fest, wie Panahi mit seinem Handy Aufnahmen vom Feuerwerk vor seinem Fenster macht, aus Langeweile, wie er angibt: „I got bored, I'm taking a film.“ Er wisse schließlich nicht, was er ansonsten tun könne (,They say, when hairdressers have nothing to do, they cut each other's hair“). Gleichzeitig ist der Regisseur frustriert angesichts der beschränkten Möglichkeiten, die ihm zur Verfügung stehen: „But what good can it do? The quality is too low. "Während der Dokumentarfilmer Mojtaba Mirtahmasb, der die Kamera auf Panahi richtet, den wirklichkeitsbeglaubigenden Auftrag der Kamera, ihre bezeugende Funktion, hochhält („I believe what matters is that it is documented“), sind es bei Panahi filmästhetische Qualitäten, die er vermisst - der Wert des Filmemachens reduziert sich für ihn eben nicht auf die dokumentarische Funktion, sondern ergibt sich aus den fingierten Geschichten, die er mit seinen Filmen erzählt.

Vor diesem Hintergrund erscheint PARdÉ/Closed CurTaIN (Reg. Jafar Panahi, Iran 2013), der sich anschließende, gemeinsam mit seinem iranischen Kollegen Kambuzia Partovi realisierte Spielfilm Panahis, als logische Weiterführung des Vorgängers, da hier der einst dokumentarische Blick auf den eigenen, von der auferlegten Berufsunfähigkeit dominierten Alltag fortgesetzt wird in einer fiktionalen Filmhandlung: Ein Drehbuchautor (gespielt von Partovi) zieht sich in ein einsames Strandhaus hinter geschlossene Vorhänge zurück, damit der Hund, den er illegalerweise hält, nicht getötet wird. ${ }^{21}$ Schon bald dringt eine junge Frau in das Haus ein, beschließt zu bleiben und verstört den Autor zunehmend. Das Parabelhafte der Filmhandlung, die Pahanis Berufsverbot und die damit verbundene Sinnkrise metaphorisiert, wird schon in den rahmenden Einstellungen des Films deutlich. Dieser beginnt und endet jeweils mit einem Blick durch die vergitterte Front des Hauses auf das weite Meer hinaus und imaginiert das Strandhaus, das nicht zufällig das reale Strandhaus Panahis ist, unverkennbar als Gefängnis.

20 This IS NOT A FILM. Reg. Jafar Panahi, Iran 2011, TC: 00:59:50-01:01:12.

21 Im Iran gelten Hunde als ,unreine‘ Tiere - das Ausführen von Hunden ist ebenso verboten wie ihr Transport in Autos. 

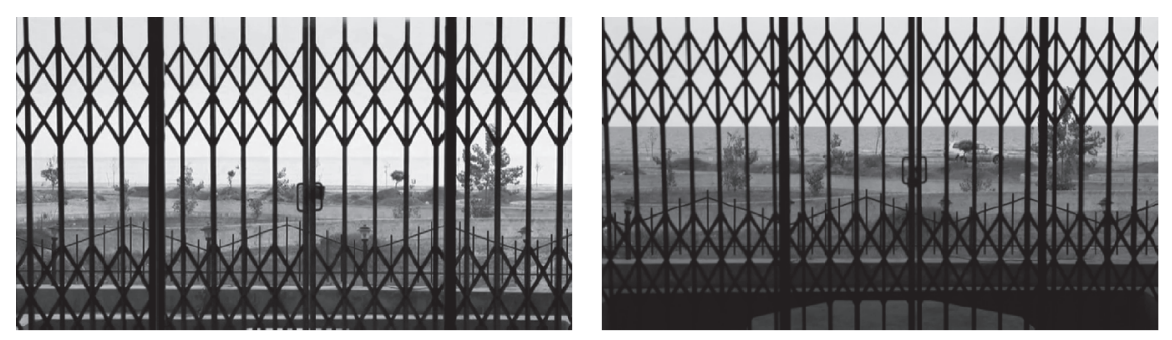

Abb. 3: Erste (TC: 00:00:01) und letzte (TC: 01:39:55) Einstellung: PARdÉ/CLOSEd CuRTAin (Reg. Jafar Panahi, Iran 2013)

In diesem Film erweist sich die Fiktionsebene als trügerisch, wenn plötzlich Jafar Panahi selbst das Haus betritt (TC: 00:56:21). Hier wandelt sich, hat Peter Uehling in der Frankfurter Rundschau ganz richtig bemerkt, der Film ,aus einer gleichnishaften Erzählung in einen mehrfach in sich gespiegelten Essay über das Filmemachen." ${ }^{22}$ Die lineare Filmerzählung wird aufgebrochen, die Grenze zwischen Einbildung, Realität und Traum auf der diegetischen Ebene in Frage gestellt und auf der extradiegetischen einmal mehr nach jener zwischen Fiktion und Wirklichkeit gefragt. Die experimentelle Filmsprache, die Panahi gemeinsam mit Partovi hier anschlägt, ist bemerkenswert nicht nur, weil sie sich deutlich vom Neorealismus seiner sonstigen Arbeiten unterscheidet, sondern weil ihm eine bild- und metaphernreiche Filmästhetik gelingt, obgleich die Rahmenbedingungen dafür eigentlich nicht gegeben sind.

\section{Fingierte Dokumentation - authentische Fiktion: TAXI (2015)}

Der 2015 veröffentlichte, als Dokufiktion rezipierte und vertriebene Film TAXI (dt. TAXI Teheran) zeigt Jafar Panahi, wie er in der Rolle eines Taxifahrers Passagiere durch Teheran fährt, die ihre jeweilige Geschichte erzählen, gefilmt von drei im Taxi montierten Kameras. Die Kameraperspektive ist, bis auf die einleitende Einstellung, fast ausnahmslos auf das Innere des Wagens beschränkt - nur an wenigen Stellen zeigt die statische Frontkamera einzelne Menschen, die aus dem Wagen aussteigen. Neben den installierten Kameras resultieren einige Filmein-

22 Uehling, Peter. „Die Geschichte soll sich nicht runden“. In: Frankfurter Rundschau vom 13.02.2013. https://www.fr.de/kultur/tv-kino/geschichte-soll-sich-nicht-runden-11277405. html (15.03.2020). 
stellungen aus Aufnahmen einer Handykamera sowie einer Digitalkamera, deren unruhige, zum Teil unscharfe Bilder nachträglich in den Film geschnitten werden.
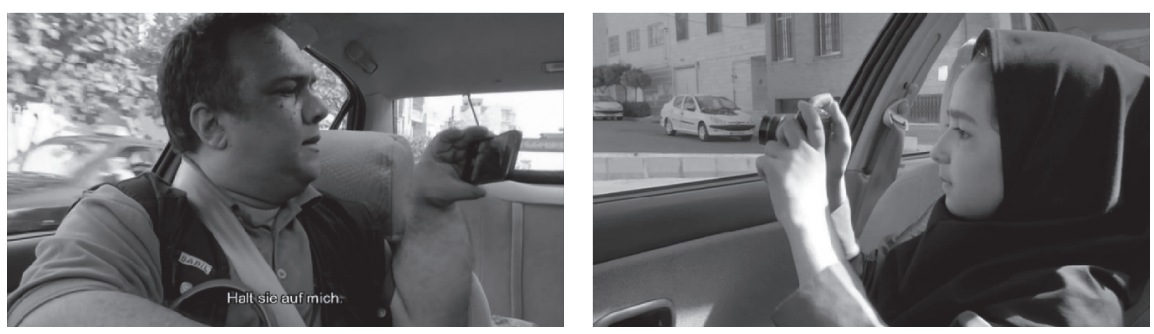

Abb. 4: Gefilmtes Filmen: Omid (00:14:02) und Hana (00:57:15)

Diese Aufnahmen gehen auf Figuren zurück, die im Film vorübergehend das Filmen übernehmen - damit kommt es zu einer diegetischen Transgression innerhalb der Erzählstruktur, die bezeichnend für den Gesamtfilm ist: Indem die Filmfiguren (die zur diegetischen Ebene des Films gehören) selbst zur Kamera greifen, avancieren sie von diegetischen Figuren zu erzählenden Instanzen der extradiegetischen Ebene - dieses Verfahren filmischer Metalepse unterläuft Grenzen zwischen Wirklichkeit und Fiktion, zwischen Dokumentation und Inszenierung.

Das Figurenensemble in Panahis Film bietet einen bemerkenswerten Querschnitt durch die Teheraner Gesellschaft. Zu Wort kommen ein Taschendieb, eine Lehrerin, der DVD-Händler Omid, Panahis Nichte Hana, zwei ältere Frauen mit einem Goldfisch, ein ehemaliger Nachbar, eine Frau an der Seite ihres schwer verletzten Ehemannes, ein Filmstudent und die iranische Anwältin und Menschenrechtsaktivistin Nasrin Sotoudeh. Die Filmerzählung lässt offen, welche Passagen dokumentarischen Charakter haben oder welche Sequenzen gänzlich fingiert sind. Vielmehr hinterfragt der Film sowohl auf der diegetischen Ebene wie auf der extradiegetischen einen normativ verstandenen Binarismus von Dokumentation und Fiktion. Die Filmfiguren selbst diskutieren wiederholt die Frage nach der sich darstellenden Wirklichkeit im Taxi und damit jene nach der „Echtheit“ der Bilder. So stellt der erste Fahrgast, der Taschendieb, angesichts der mangelnden Ortskenntnis seines Fahrers fest: „Sie können doch kein Taxifahrer sein. Das kann doch nicht wahr sein“ (TAXI, 00:06:32), während Omid, der illegal mit DVDs handelt, Panahi die Rolle des Taxifahrers nicht im Ansatz abnimmt: „Erzählen Sie mir nicht, dass Sie nur Taxi fahren.“ (TAXI, 00:10:38) Mehrfach durchbrechen diese Filmfiguren die vierte Wand, wenn sie in einer äußeren Metalepse die im Auto positionierten Kameras thematisieren und dann direkt in diese schauen. Etwa der Taschendieb, der die Kamera entdeckt und glaubt, dass diese eine Schutzmaß- 

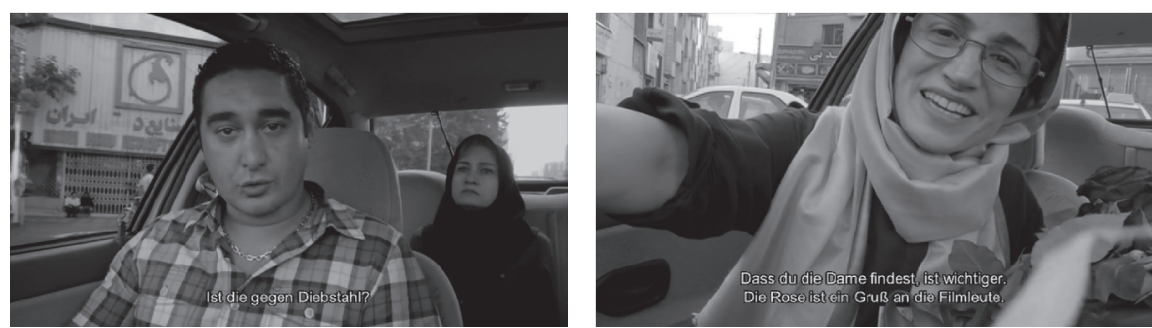

Abb. 5: Filmische Metalepsen: TAXI (00:03:15; 01:10:24)

nahme gegen Diebstahl sei, oder die Menschenrechtsanwältin, die der Kamera eine Rose schenkt, als „Gruß an die Filmleute“, denn diese seien schließlich „ganz wunderbare Menschen“ (TAxI, 01:10:17).

Eine letzte Transgression beendet den Film: Während Panahi das Taxi erstmalig verlässt, erstürmt ein Motorradfahrer das Auto und raubt die Kamera, allerdings, wie er seinem Begleiter zuruft, ohne ihre Speicherkarte.

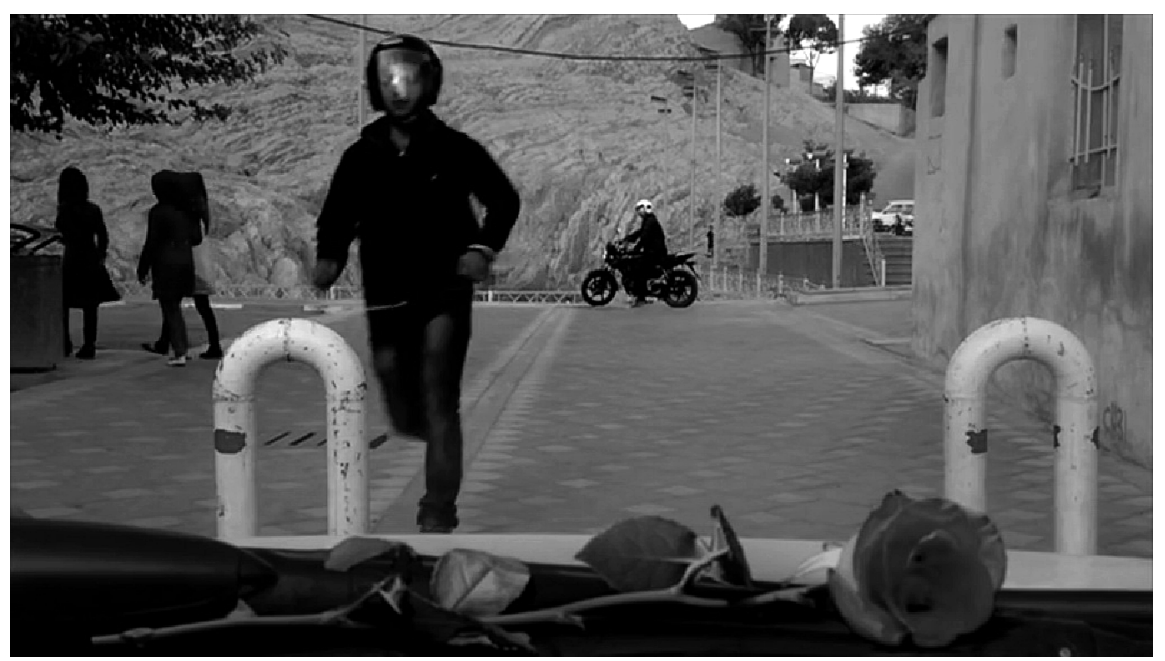

Abb. 6: TAXI (01:16:47)

Der gefilmte Raub auf der diegetischen Ebene beendet zugleich die Filmerzählung auf der visuellen Ebene, die mit einer Schwarzblende schließt - auf der auditiven Ebene ist nur noch die Stimme des Einbrechers im Off zu hören, der mit der Kamera, aber ohne Speicherkarte das Wageninnere verlässt.

Der Schauplatz des Films, das Taxi, spielt eine, auch für die Frage nach filmischen Authentifizierungsstrategien, relevante Rolle - wie für Panahis Filme Fahr- 
zeuge generell wichtig sind. OFFSIDE etwa beginnt mit einer Einstellung im Auto und spielt im Anschluss daran unter anderem in einem Linienbus, ebenso wie der Spielfilm Ayneh/Der SpIEgel. Der aktuellste Film, Se RoKh/Drei GeSichter, in dem Panahi an der Seite der Schauspielerin Behnaz Jafari zu einer Reise in den Nordwesten Irans aufbricht, um dort den vermeintlichen Selbstmord eines jungen Mädchens aufzuklären, wurde gar als Roadmovie wahrgenommen und vermarktet. ${ }^{23}$ Die Wahl bewegter Fahrzeuge als filmische Schauplätze ist vor allem eine pragmatische, die den politischen Umständen im Iran geschuldet ist und diese zugleich offensichtlich werden lässt. Gilt das Auto grundsätzlich als Symbol der Freiheit (wie es das Roadmovie genretypisch inszeniert), wird in der Gegenwart des Iran aus der symbolischen eine tatsächliche Freiheit. Das Taxi, ein in Teheran günstiges und äußerst populäres Verkehrsmittel, stellt einen gleichermaßen intimen wie geschützten Raum zur Verfügung, in der das Individuum der staatlichen Dauerüberwachung und dem kollektiven Misstrauen zumindest für eine kurze Zeit entkommt. „Ein Teheraner Taxi“, so hat das Ulrich Ladurner in der ZEIT pointiert, ,ist eine Freiheitsbox auf Rädern. “24 Eine Freiheitsbox, die in ihrer Anonymität Formen der Authentizität und Geschichten möglich macht, die nicht danach streben, die Wirklichkeit Teherans zu zeigen, sondern die vermeintliche Opposition von Wahrheit und Fiktion gerade im Kontext realer politischer Umstände zu hinterfragen.

Dass es in Panahis TAXI - anders als im Roadmovie - nicht um die Geschwindigkeit oder die Bewegung durch eine bestimmte Landschaft geht, macht schon die erste, außerordentlich lange, statische Einstellung des Films deutlich (vgl. TAXI, 00:00:00-00:01:25). Aus einem unbewegten Auto heraus richtet sich der Blick auf die Straßen Teherans - erst nach gut einer Minute Filmzeit fährt das Taxi langsam an, um nach wenigen Metern wieder zu halten und den ersten Gast einsteigen zu lassen. Die Filmerzählung definiert sich, im Unterschied zum Roadmovie, nicht über den Raum, den sich das Auto erfährt, sondern über die Beobachtungen, die im Inneren des Autos möglich werden. Der begrenzte Raum des Taxis unterscheidet sich dabei entscheidend von den geschlossenen Räumen, die Panahi etwa in CLOSED CURTAINS als Gefängnis des Künstlers inszeniert. Im Gegensatz zu den dortigen klaustrophobisch anmutenden Räumen bleibt der Innenraum des Taxis trotz der Enge in ständiger Bewegung - und angesichts der fluktuierenden Belegschaft ein belebter Raum des Austausches, der im Dualismus von temporärer Intimität und gleichzeitiger Anonymität entsteht. André Bazin hat das Au-

23 SE RoKh/Drei Gesichter, Reg. Jafar Panahi, Iran 2018.

24 Ladurner, Ulrich. „Frei für einen Augenblick“. In: Die Zeit vom 23.07.2015. https://www.zeit. de/2015/30/taxi-teheran-film-jafar-panahis (15.03.2020). 
to bzw. das Fahrzeug als filmischen Schauplatz in seinem wirkmächtigen Essay Der kinematografische Realismus und die italienische Schule der Befreiung als Qualitätsmerkmal neorealistischer Filme hervorgehoben. Bazin spricht von Szenen, „die eine ganz besondere Intensität des Schauplatzes und der Menschen aufweisen“, gerade aufgrund des „natürlichen Verhalten[s] aller Personen“, die hier auf engstem Raum zusammenkommen. ${ }^{25}$ So spiegelt das Auto als Schauplatz einmal mehr den Einfluss des italienischen Neorealismus auf das iranische Kino der Gegenwart - der iranische Kultur- und Kommunikationswissenschaftler Hamid Naficy spricht gar von einem „Neorealism iranian style“. ${ }^{26}$ Naficy erkennt Analogien zwischen dem neorealistischen Erzählen italienischer Herkunft und seinen iranischen Nachfolgern dort, wo sich die Filme beider Provenienzen als geographisch und zeitlich gebunden erweisen und ähnlichen formalen Regeln folgen, die auch in Panahis Filmen zu finden sind: Lange Einstellungen, Shooting on Location, unsichtbare Schnitte, gegenwartsgebundene Themen, offene Enden, Protagonisten aus der Arbeiterklasse, dargestellte Sozio- und Dialekte und eine zumindest implizite Sozialkritik. ${ }^{27}$ Die Analogien zwischen dem iranischen Kino der Gegenwart und dem italienischen Neorealismo, die insbesondere in einer gemeinsamen Form- und Bildsprache begründet liegen, sollen, so argumentiert auch Naficy, nicht über die Grenzen dieses Vergleichs hinwegtäuschen. Iranische Filme, allen voran das Spätwerk Kiarostamis, vermischen, so betont Naficy, ausdrücklich Illusion und Wirklichkeit und erzeugen bewusst Unsicherheit und Ambiguität - „a far cry from the classic neorealist concern and style." 28

Und so bekennt sich Panahi mit seiner erkennbar hybriden, Elemente des Spiel- wie des Dokumentarfilms aufgreifenden Ästhetik in deutlicher Erweiterung des italienischen Neorealismo zu einer spezifisch iranischen Filmsprache, die unter dem Begriff „New Wave“ firmiert und eine Filmtradition bezeichnet, die schon vor der iranischen Revolution 1979 beginnt. Das iranische New Wave Cinema versteht sich in der Nachfolge des italienischen Neorealismo - Jafar Panahi ist unter seinen iranischen Kollegen keine Ausnahme, wenn er wiederholt konstatiert, dass kein Film ihn so sehr beeinflusst habe wie Vittorio de Sicas Ladri di Biciclet-

25 Bazin, André. Was ist Kino. Bausteine zur Theorie des Films. Köln: DuMont Schauberg 1975, 153.

26 Naficy, Hamid. „Neoralism iranian style“. In: Global Neorealism. The Transnational History of a Film Style. Hg. Saverio Giovacchini, Robert Sklar. Jackson: University Press of Mississippi 2012, 226-239.

27 Naficy: „Neoralism iranian style“, 226. Naficy geht in seinem Beitrag allerdings nicht explizit auf Panahi ein, sondern berücksichtig allen voran dessen ,Lehrmeister“ Kiarostami.

28 Naficy: „Neoralism iranian style“, 238. 
te. ${ }^{29}$ Die neorealistischen Elemente in Panahis Filmen, seine Leidenschaft für das italienische Kino der 1950er Jahre und die Vorliebe für das Auto als Drehort sind dabei kaum ohne den Einfluss von Abbas Kiarostami auf das Selbstverständnis Panahis als Filmemacher nachvollziehbar. Abbas Kiarostami, heißt es in einem Nachruf von Wenke Husmann in der Zeit, sah die Welt durch eine Autoscheibe und machte „große Kunst“ daraus. ${ }^{30}$

TAXI macht Kunst weniger aus den Geschichten jenseits der Autoscheibe als vielmehr aus denen im geschützten Raum, der sie begrenzt. Diese Geschichten sind gleichermaßen authentisch wie sonderbar: Etwa die des schwer verletzten Mannes, der vor Zeugen seinen letzten Willen aufs Handy diktiert, damit seine ansonsten rechtlose Ehefrau im Todesfall nicht durch die eigenen Brüder ums Erbe gebracht wird. Oder die beiden streitenden Frauen, die unbedingt bis 12 Uhr mittags zu einer heiligen Quelle gefahren werden müssen, um dort ihre Goldfische auszusetzen. Oder die eines ehemaligen Nachbarn, der von einem befreundeten Ehepaar zusammengeschlagen und ausgeraubt wird und das Überwachungsvideo des Überfalls Panahi zeigt, der die Aufnahme angesichts des Berufsverbots zwar nicht verwerten könne - aber vielleicht ja ein anderer Filmemacher? Natürlich wird der dokumentarische Wert dieses Überwachungsfilms gleich wieder in Frage gestellt, nicht nur, weil der Täter darauf vermummt und nicht zu erkennen ist. Vielmehr handelt es sich beim Täter in Wirklichkeit um jenen Kellner, der, so informiert der Nachbar den entgeisterten Panahi, ihnen soeben einen Saft ans Auto gebracht habe (vgl. TAxi, 00:50:25).

Die wirklichkeitsbeglaubigende Funktion des Films wird bereits hier selbstreflexiv subvertiert - noch stärker ist dies der Fall, wenn Panahis Nichte Hana ins Taxi einsteigt. Die Nichte ist eine Schlüsselfigur für das metafiktionale Potenzial des Films. Hana soll mit ihrem Handy einen Film für die Schule drehen - und ist nun auf der Suche nach einer geeigneten Geschichte. Das Problem: Der Film soll „vorzeigbar“ sein, die Wirklichkeit zeigen und wahr sein, unterliegt dabei aber strikten Regeln, die sie ihrem Onkel auflistet: „Keine Berührung zwischen Mann und Frau, keine Schwarzmalerei, keine Gewaltdarstellung, keine Krawatten für die positiven Figuren, keine persischen Namen für die positiven Figuren. Besser man verwendet die Namen der heiligen Imame“ (TAXI, 00:54:00-00:55:50). Den strikten Regeln dieses Filmunterrichts widersetzen sich sämtliche Geschichten,

29 Vgl. Ruberto, Laura E./Wilson, Kristi M. „Italian Neorealism: Quotidian Storytelling and Transnational Horizons“. In: A Companion to Italian Cinema. Hg. Frank Burke. Chichester/West Sussex: Wiley-Blackwell 2017, 139-157, hier 151.

30 Husmann, Wenke. „Abbas Kiarostami. Er setzte sich über die Realität hinweg“. In: Zeit online vom 05.07.2016. https://www.zeit.de/kultur/film/2016-07/abbas-kiarostami-filmemacheriran-nachruf (20.03.2020). 
die im Taxi erzählt und aufgezeichnet werden, in denen es eben keine Schurken mit Krawatte und keine Helden gibt, die den Namen der heiligen Imame tragen. Im Taxi ihres Onkels begreift Hana, dass die Wirklichkeit, die sie filmen soll, jene Geschichten, die man vor ihr verlangt, gerade nicht hergibt. Und dass Wahrheit und Wirklichkeit keine Synonyme darstellen. Dieser Erkenntnisprozess Hanas (der sich mit jenem der Zuschauer^innen überschneidet) verdankt sich vor allem dem letzten Fahrgast, der ins Taxi steigt: Es handelt sich dabei um Nasrin Sotoudeh, eine im Film wie im wahren Leben Anwältin und Menschenrechtsaktivistin, die im Iran Regimegegner`innen und unterdrückte Frauen verteidigt und im Gespräch mit Panahi einen kurzen Einblick in ihren Alltag gewährt. Diese Passage ist angesichts der politischen Härte und Willkür, von der Soutoudeh berichtet, der Undurchschaubarkeit juristischer Entscheidungen und der ständigen Gefahr, im Gefängnis zu landen, sicherlich die düsterste des Films. ${ }^{31}$ Der ungebrochene Optimismus und die kompromisslose Lebenszugewandtheit der Figur Sotoudehs, die sich den widrigen politischen Bedingungen mit einem Strauß roter Rosen in den Weg stellt, halten die erzählerische Beschwingtheit jedoch bis zuletzt aufrecht. Nach Sotoudehs verstörendem Bericht zur Lage in den Gefängnissen Teherans und den stets drohenden Instrumenten politischer Willkür sind nicht nur bei Hana die Konzepte Wirklichkeit, Wahrheit und Fiktion endgültig ins Wanken geraten. Was denn der Begriff „Schwarzmalerei“ eigentlich bedeute, will die Nichte von Panahi, dem Taxifahrer, wissen, der sie wiederum auf den Inhalt ihrer Schulhefte und ihre Lehrerin verweist. Die aber, entgegnet Hana, könne offenbar nicht helfen:

Aber was unsere Lehrerin dazu erklärt hat, hab ich nicht kapiert. Sie kann nicht gut erklären. Wir sollen die Realität zeigen, nichts Unwirkliches. Und wenn die Realität unschön und düster ist, sollen wir sie nicht zeigen. Ich verstehe echt nicht so richtig, was die Realität von der Unwirklichkeit unterscheidet. Das ist mir zu hoch. (TAXI, 01:11:57-1:12:30)

\section{Hybride Ästhetik}

Spätestens hier wird deutlich: Panahis Film geht es keinesfalls darum, das im Raum des Taxis Gesagte als gültige Wahrheit zu behaupten und damit einen plumpen Gegensatz zwischen filmischer Wahrheit und politischer Lüge aufzuspannen,

\footnotetext{
31 Nasrin Sotoudeh verbüßt inzwischen eine 33-jährige Haftstrafe im berüchtigten iranischen Frauengefängnis Ghartschak. Am 03.12.2020 wurde sie für ihr unerschrockenes Engagement zur Förderung politischer Freiheiten und der Menschenrechte mit dem sogenannten Alternativen Nobelpreis (Right Livelihood Award) ausgezeichnet.
} 
sondern den Begriff der Wahrheit wie der Wirklichkeit bzw. den vermeintlichen Binarismus von Fiktion und Wirklichkeit inhaltlich wie formal-stilistisch ebenso zu hinterfragen wie jenen zwischen den Gattungen Dokumentar- und Spielfilm. Damit lässt er sich als „,ästhetische Intervention der Gattungslogik“ verstehen so hat der Medienwissenschaftler Philipp Blum versucht, das kritische Potenzial hybrider Filmerzählungen fiktions- und filmtheoretisch einzufangen:

Hybridisierungen des Dokumentarischen [...] sind also nicht einfach Spielfilme im Gewand des Dokumentarischen, gefälschte Dokumentationen oder hyperreale Spielfilme; vielmehr geben ihre Erscheinungen Anlass, über Film und seine Medialität zu reflektieren und dies jenseits überkommener Dichotomien von Beweis und Illusion. ${ }^{32}$

Panahis Film agiert dort im kritischen Sinne ,hybrid', wo er mit seinen metaleptischen Transgressionen für eine Beobachtung zweiter Ordnung sorgt und das filmische Erzählen ebenso in den Blick nimmt wie den Regisseur Jafar Panahi als erzählte und nicht nur erzählende Figur. In diesem hybriden Selbstverständnis, das sich gerade über die Unentschiedenheit zwischen dokumentarischem Zeigen und filmisch-fiktionalem Erzählen definiert, verbirgt sich, und das ist vielleicht die einzig eindeutige Botschaft des Films, eine Liebeserklärung an den Film und an das Kino - und seine spezifischen medialen Möglichkeiten, Geschichten im Grenzraum von Fakt und Fiktion zu erzählen. Programmatisch ist vor diesem Hintergrund das den Film prägende dichte Netz an Bezügen zum Filmbetrieb und zur Filmgeschichte: Dazu zählen die zahlreichen Gespräche über Filme, etwa mit Omid, dem DVD-Händler, der seine illegale Tätigkeit zur Kulturarbeit stilisiert, mit Hana, der Nichte, oder mit dem jungen Filmstudenten, der von Panahi wissen will, wo er die Geschichte für seinen Abschlussfilm an der Filmakademie finden soll. Diese filmischen Anspielungen öffnen eine letzte selbstreferenzielle Ebene, die den Authentifizierungsstrategien eines Dokumentarfilms entgegensteuert. Allen voran die intertextuellen Verweise auf das Gesamtwerk Panahis (wenn sie denn entschlüsselt werden) relativieren den Eindruck von Unmittelbarkeit der Geschehnisse und Gespräche im Taxi. Zum Beispiel: Der Goldfisch, den die beiden

32 Blum: „Doku-Fiktionen“, 141. In seiner 2017 erschienenen Dissertation erweitert Blum seine Auseinandersetzung mit hybriden Filmformaten und schlägt eine Ästhetik des „queeren Films“ vor, wobei er queer im Anschluss an die politische Emanzipation des ursprünglich negativ konnotierten Begriffes im Kontext der Gender und Queer Studies als Gattungsmerkmal etabliert, das nicht nur die hybride filmische Form, sondern damit verbundene Prozesse des Widerstandes und der Subversion meint. Diese Übertragung eines kulturtheoretisch so eindeutig besetzten Begriffs scheint mir recht problematisch, weil sie den explizit politischen Gehalt des Queer-Begriffes zu relativieren droht. Vgl. Blum, Philipp. Experimente zwischen Dokumentar- und Spielfilm. Zu Theorie und Praxis eines ästhetisch ,queeren“ Filmensembles. Marburg: Schüren 2017. 
Frauen pünktlich zu einer Heiligen Quelle bringen möchten, verweist zurück auf den Debütfilm Panahis, in dem sich die Protagonistin sehnlichst wünscht, einen Goldfisch zu besitzen. Die Anwältin erzählt von einer Mandantin, die inhaftiert ist, weil sie heimlich versucht hat, einer Sportveranstaltung beizuwohnen (wie in Panahis Film OfFSIDE), und Hana, die Nichte, droht ihrem unpünktlichen Onkel, dass sie ja auch alleine den Weg nach Hause finden könne - wie Mina, die Protagonistin aus AYNEH. Dort, wo die fiktive Wirklichkeit des Films nicht mehr auf die afilmische Realität, sondern auf andere filmische Fiktionen verweist, ist nicht mehr das Dokumentarische, sondern das Fiktionale beglaubigend. Vielleicht, so scheinen Panahis Filme zu behaupten, weil ihre Qualität gerade darin liegt, authentische Geschichten zu erzählen, die wahr sein können, aber sicher nicht müssen.

\section{Filmografie}

Ayneh/Der Spiegel. Reg. Jafar Panahi. Iran 1997.

Badkonake Sefid/Der weisse Ballon. Reg. Jafar Panahi. Iran 1995.

DAYEReh/DeR KReIS. Reg. Jafar Panahi. Iran/Schweiz/Italien 2000.

OFFSIDE. Reg. Jafar Panahi. Iran 2006.

Pardé/Closed Curtain. Reg. Jafar Panahi. Iran 2013.

SE ROKH/DREI Gesichter. Reg. Jafar Panahi. Iran 2018.

TAXI. Reg. Jafar Panahi. Iran 2015.

THIS IS NOT A FILM. Reg. Jafar Panahi. Iran 2011.

\section{Literatur}

Bazin, André. Was ist Kino. Bausteine zur Theorie des Films. Köln: DuMont Schauberg 1975.

Blum, Philipp. „Doku-Fiktionen. Filme auf der Grenze zwischen Fiktion und Non-Fiktion als ästhetische Interventionen der Gattungslogik“. In: MEDIENwissenschaft 2 (2013), 130-144.

Blum, Philipp. Experimente zwischen Dokumentar- und Spielfilm. Zu Theorie und Praxis eines ästhetisch, queeren' Filmensembles. Marburg: Schüren 2017.

Fludernik, Monika/Falkenhayner, Nicole/Steiner, Julia. „Einleitung“. In: Faktuales und Fiktionales Erzählen. Schriftenreihe des Graduiertenkollegs 1767. Hg. Monika Fludernik, Nicole Falkenhayner, Julia Steiner. Würzburg: Ergon 2015, 7-22.

Habermas, Jürgen. „Erläuterungen zum Begriff des kommunikativen Handelns“. In: Vorstudien und Ergänzungen zur Theorie des kommunikativen Handelns. Hg. Jürgen Habermas. Frankfurt a. M.: Suhrkamp 1995 [1982], 571-606.

Hißnauer, Christian. „MöglichkeitsSPIELräume. Fiktion als dokumentarische Methode. Anmerkungen zur Semio-Pragmatik Fiktiver Dokumentationen “. In: MEDIENwissenschaft 1 (2010), 17-28. 
Hohenberger, Eva. Die Wirklichkeit des Films. Dokumentarfilm. Ethnographischer Film. Jean Rouch. Hildesheim/Zürich/New York: Olms 1988.

Husmann, Wenke. „Abbas Kiarostami. Er setzte sich über die Realität hinweg“. In: Zeit online vom 05.07.2016. https://www.zeit.de/kultur/film/2016-07/abbas-kiarostamifilmemacher-iran-nachruf (20.03.2020).

Klingenmaier, Thomas. „Die Gewitztheit des Verfolgten“. In: Stuttgarter Zeitung vom 23.07.2015. https://www.stuttgarter-zeitung.de/inhalt.untergrundkino-aus-dem-irantaxi-teheran-die-gewitztheit-des-verfolgten.afd26a5a-6151-4e3b-b4bf-26c3eadc43ce. html (15.03.2020).

Ladurner, Ulrich. „Frei für einen Augenblick“. In: Die Zeit vom 23.07.2015. https://www.zeit.de/ 2015/30/taxi-teheran-film-jafar-panahis (15.03.2020).

Martínez, Matías/Scheffel, Michael. Einführung in die Erzähltheorie. 10., überarb. und aktual. Auflage. München: C.H.Beck 2016.

Mundhenke, Florian. Zwischen Dokumentar- und Spielfilm. Zur Repräsentation und Rezeption von Hybrid-Formen. Wiesbaden: Springer 2017.

Naficy, Hamid. „Neoralism iranian style“. In: Global Neorealism. The Transnational History of a Film Style. Hg. Saverio Giovacchini, Robert Sklar. Jackson: University Press of Mississippi 2012, 226-239.

Nicodemus, Katja. „Der Freie“. In: Die Zeit vom 07.07.2006. https://www.zeit.de/2016/29/ nachruf-abbas-kiarostami (24.03.2020).

Nord, Christian: „Eine Irrfahrt ohne Abspann“. In: taz vom 22.07.2015. https://taz.de/FilmstartTaxi-Teheran/ (15.02.2020).

Odin, Roger. „Dokumentarischer Film - dokumentarisierende Lektüre“. In: Bilder des Wirklichen. Texte zur Theorie des Dokumentarfilms. Hg. Eva Hohenberger. 3. Auflage. Berlin: Vorwerk 2006, 259-275.

Ruberto, Laura E./Wilson, Kristi M. „Italian Neorealism: Quotidian Storytelling and Transnational Horizons“. In: A Companion to Italian Cinema. Hg. Frank Burke. Chichester/West Sussex: Wiley-Blackwell 2017, 139-157.

Souriau, Etienne. „Die Struktur des filmischen Universums und das Vokabular der Filmologie“ [= La structure de l'univers filmique et le vocabulaire de la filmologie 1951]. In: montage AV. Zeitschrift für Theorie und Geschichte audiovisueller Kommunikation, 6.2 (1997), 140-157.

Uehling, Peter. „Die Geschichte soll sich nicht runden“. In: Frankfurter Rundschau vom 13.02.2013. https://www.fr.de/kultur/tv-kino/geschichte-soll-sich-nicht-runden11277405.html (15.03.2020).

Weixler, Antonius. „Authentisches erzählen - authentisches Erzählen. Über Authentizität als Zuschreibungsphänomen und Pakt“. In: Authentisches erzählen. Produktion, Narration, Rezeption. Hg. Antonius Weixler. Berlin/Boston: De Gruyter 2012, 1-32. 
